\title{
Precision Electroweak Measurements at the LHC and Tevatron
}

\author{
Marco Pieri* on behalf of the ATLAS, CDF, CMS, D0, and LHCb Collaborations \\ UC San Diego, United States \\ E-mail: marco.pieriecern.ch
}

\begin{abstract}
Various electroweak precision measurements are performed at the hadron colliders Tevatron and LHC. The main ones regard the masses of the $W$ boson, top quark and Higgs boson, and $\sin ^{2}$ eff. This talk covers the latter and also other measurements carried out using Drell-Yan decays. The results of the $\sin ^{2}$ eff measurements have an accuracy of approximately twice that reached at LEP and SLD. The LEP and SLD accuracy is expected to be approached with Run 2 and Run 3 data and surpassed with high-luminosity LHC data. Other measurements reported are the differential distributions and the full set of decay angular coefficient in Drell-Yan events.
\end{abstract}

Sixth Annual Conference on Large Hadron Collider Physics (LHCP2018)

4-9 June 2018

Bologna, Italy

\footnotetext{
* Speaker.
} 


\section{Introduction}

The LHC and other hadron colliders measure many parameters of the Standard Model (SM) and they give very important inputs to the global SM fits [1]. The most relevant SM parameters which are discussed in other talks at the conference are: the $W$ boson mass $m_{W}$, measured at the Tevatron and at the LHC by ATLAS which can be compared to the SM prediction from the Z boson mass $m_{Z}$ and electroweak precision measurements; the top quark mass $m_{\mathrm{t}}$, which affects radiative corrections and is precisely measured at the LHC and Tevatron; the Higgs boson mass $m_{H}$, which affects radiative corrections and is precisely measured at the LHC; Higgs boson couplings, also entering the picture through radiative correction, are studied at the LHC. In this presentation the measurements of the effective leptonic weak mixing angle $\left(\sin ^{2} \theta_{\text {eff }}^{\ell}\right)$ and other studies of $\mathrm{W}$ and $\mathrm{Z}$ decays carried out at hadron colliders are reviewed. These studies are based on large samples of $\gamma^{*} / Z$ decays to electrons and muons, referred in the following as $Z$ decays, collected at the Tevatron and during the Run 1 of the LHC.
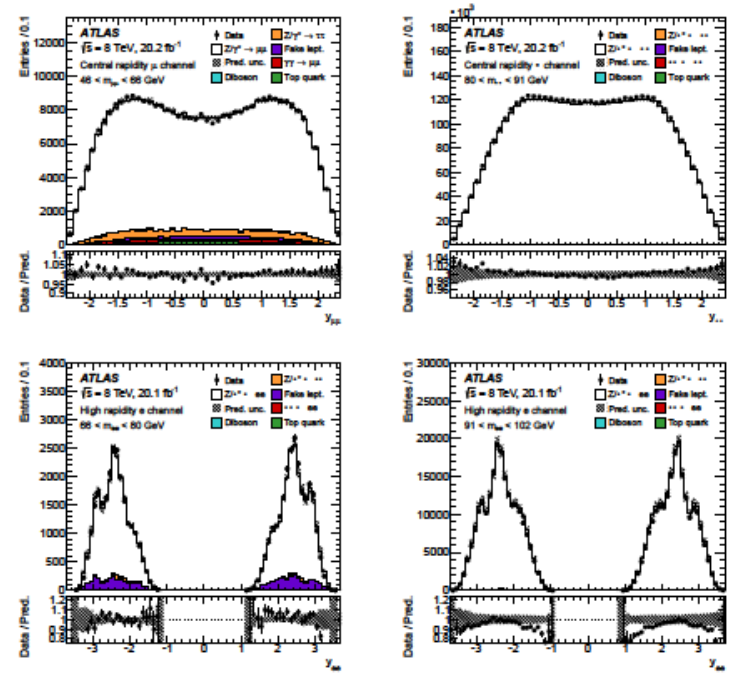

Figure 1: $Z$ boson rapidity in $Z$ decays into muons (top plots) and electrons with one high rapidity electron (bottom plots). The two left plots show events with dilepton mass below the $Z$ peak while the right two plots are for masses around the $Z$ peak [2].

The interest of measuring $\sin ^{2} \theta_{\mathrm{W}}$ is twofold: on one side it provides an indirect measurement of $m_{W}$, through the relation:

$$
\sin ^{2} \theta_{\mathrm{W}}=1-\frac{m_{W}^{2}}{m_{Z}^{2}}
$$

and on the other it may help to disentangle the well known discrepancy of approximately $3 \sigma$ between the most precise measurements which were obtained at LEP and SLD [3]. Different experiments use $Z$ boson decays into muon pairs and whenever possible also into electron pairs. The standard selection of $Z$ decays requires both leptons to have a pseudo-rapidity $|\eta|<2.4$ but ATLAS also uses electron pairs with a forward electron which does not traverse the tracker and is identified and measured in the calorimeters. Figure 1 shows the rapidity spectrum of selected decays in various dilepton mass bins. The background level is very small in all cases and negligible for central events. 


\section{Measurement of $\sin ^{2} \theta_{\mathrm{eff}}^{\ell}$}

The most precise measurement of $\sin ^{2} \theta_{\mathrm{eff}}^{\ell}$ at hadron colliders is based the forward-backward asymmetry $\mathrm{A}_{\mathrm{FB}}$ in Drell-Yan $q q \rightarrow \ell^{+} \ell^{-}$events, where $\ell$ stands for muon $(\mu)$ or electron $(e)$. The forward-backward asymmetry is defined using the angle $\theta^{*}$ between the outcoming lepton and the incoming quark in the Collins-Soper reference frame. A needed ingredient to define $\theta^{*}$ is the direction of the incoming quark. At the Tevatron, which is a $p \bar{p}$ machine, the direction the (anti)quark is almost always the same as that of the (anti)proton, as valence quarks dominate. At the LHC, a $p p$ collider, the direction of the quark can be approximated to be the same as the longitudinal component of the $Z$ boson momentum because antiquarks originate from the sea and tend to have lower momentum than quarks which are predominantly valence quarks. Given the uncertainties in the quark assignment at the LHC, which are largest for central events, the observed asymmetry is reduced, compared to the true one and this effect is referred as dilution. Figure 2 shows the true asymmetry and the diluted asymmetry at the LHC in bins of $Z$ boson rapidity. As anticipated, when the $Z$ boson has no longitudinal momentum, the observed asymmetry becomes 0 as it becomes impossible to identify the quark direction. The differential cross section at leading
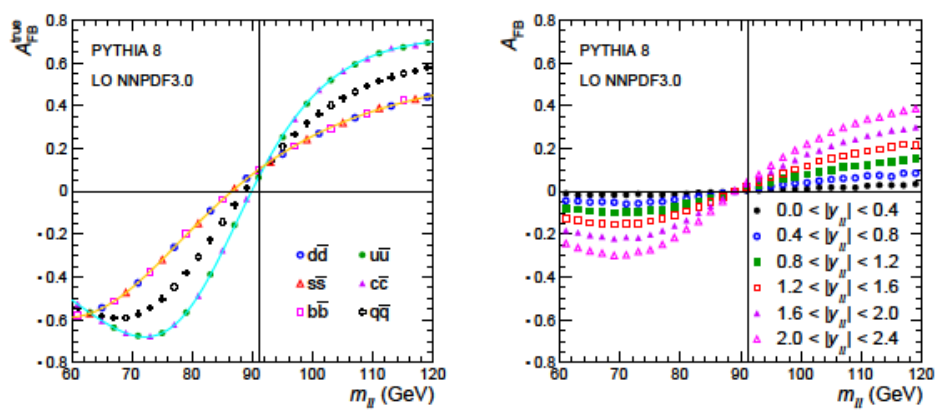

Figure 2: Left: true $A_{F B}$ for the different flavours of interacting quarks. Right: $A_{F B}$ after dilution in different ranges of $y[4]$.

order is:

$$
\frac{\mathrm{d} \sigma}{\mathrm{d}\left(\cos \theta^{*}\right)} \propto 1+\cos ^{2} \theta^{*}+A_{4} \cos \theta^{*},
$$

where $\theta^{*}$ is the polar angle of the negative lepton in the Collins-Soper frame of the dilepton system and $A_{F B}$ is defined as:

$$
\mathrm{A}_{\mathrm{FB}}=\frac{3}{8} A_{4}=\frac{\sigma_{\mathrm{F}}-\sigma_{\mathrm{B}}}{\sigma_{\mathrm{F}}+\sigma_{\mathrm{B}}},
$$

where $\sigma_{\mathrm{F}}$ and $\sigma_{\mathrm{B}}$ are the cross sections for leptons in the forward and backward hemispheres in terms of $\theta^{*}$.

$A_{F B}$ originates from the interference between vector and axial couplings and is therefore sensitive to:

$$
\begin{array}{r}
v_{\mathrm{f}}=T_{3}^{\mathrm{f}}-2 Q_{\mathrm{f}} \sin ^{2} \theta_{W}, \\
a_{\mathrm{f}}=T_{3}^{\mathrm{f}},
\end{array}
$$

with $Q_{\mathrm{f}}$ and $T_{3}^{\mathrm{f}}$ being the charge and the third component of the weak isospin of the fermion, respectively. The weak mixing angle $\sin ^{2} \theta_{W}$ is related to the masses of the $W$ and $Z$ bosons 
through the relation in Eq. 1.1. Equation 2.1 is valid at leading order and is affected by electroweak radiative corrections. In the improved Born approximation the effective mixing angle, $\sin ^{2}{ }_{\text {eff }}$, is defined, which absorbs some of the higher-order corrections.

\section{Measurement of $\sin ^{2}$ eff at the Tevatron}

The first measurements of $\sin ^{2}$ eff at hadron colliders have been carried out at the Tevatron. Tevatron is a $p \bar{p}$ collider and this is ideal for this measurement as the directions of the interacting quark and anti-quark are known with great accuracy. Both CDF and D0 measure $\mathrm{A}_{\mathrm{FB}}$ separately for muons and electrons, using template fits [5, 6, 7]. Furthermore, CDF devised methods to extract the asymmetry that are now also used in CMS: they used event weighting [11] to measure the asymmetry using cos information and they weight parton distribution function (PDF) replicas to also constrain PDFs with data [12]. Figure 3 shows both the folded and unfolded asymmetry distributions as function of the dilepton mass for $\mathrm{CDF}$, based on the full dataset, corresponding to $9 \mathrm{fb}{ }^{1}$.
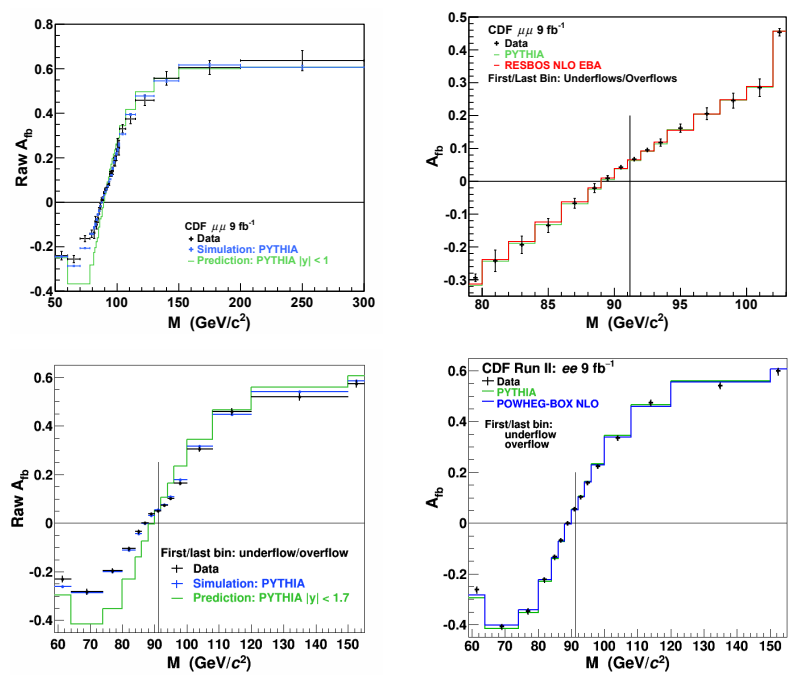

Figure 3: Measured $\mathrm{A}_{\mathrm{FB}}$ asymmetry at $\mathrm{CDF}$ as function of $m$ for muons (top) and electrons (bottom). The two plots on the left show the raw measurement while those on the right the unfolded ones [5].

Recently a full Tevatron combination has been carried out [8] and the results are:

$$
\begin{array}{llll}
\sin ^{2}{ }_{\text {eff }}=023148 & 000027 & 000018=023148 & 000033 \\
\sin ^{2}{ }_{\mathrm{w}}=022324 & 000026 & 000019=022324 & 000033
\end{array}
$$

where the largest contribution to the uncertainty is statistical and the measurement of $\sin ^{2} \mathrm{w}$ has been obtained within the on-shell scheme, using ZFITTER [9].

\section{Measurement of $\sin ^{2}$ eff at $\mathrm{LHCb}$}

LHCb measured $\sin ^{2}$ eff using the full Run 1 data, corresponding to an integrated luminosity of approximately $3 \mathrm{fb}^{1}$ [10]. Given the configuration of $\mathrm{LHCb}$, which is a single arm detector covering large values of rapidities, the measurement is carried out at large $\mathrm{Z}$ rapidity: $20<<45$, 
reducing the dilution and largely increasing the accuracy of the estimation of the quark direction, compared to ATLAS and CMS. The measured asymmetry is shown in Fig. 4 and the result of the measurement is $\sin ^{2} \theta_{\text {eff }}^{\ell}=0.23142 \pm 0.00073 \pm 0.00052 \pm 0.00056$, where the first uncertainty is statistical, the second systematic, and the third related to PDF uncertainties. The sensitivity per unit of integrated luminosity is larger than that at CMS or ATLAS and this is mainly due to the smaller dilution.
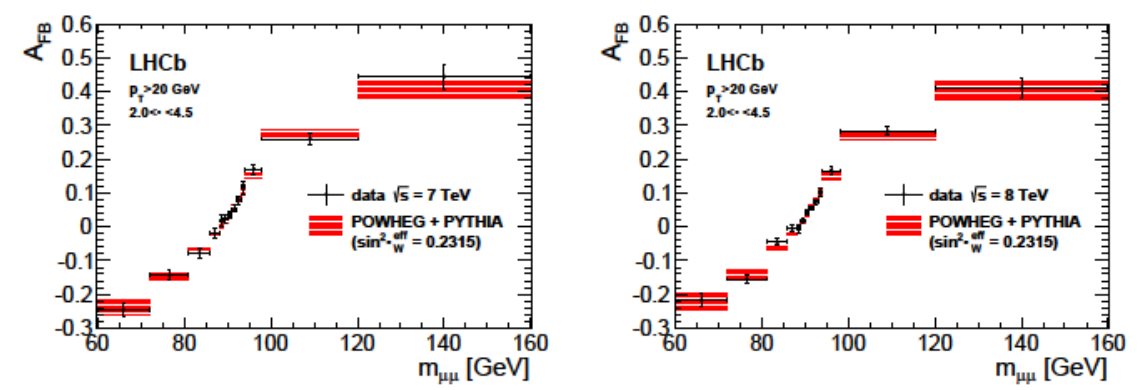

Figure 4: Measured $\mathrm{A}_{\mathrm{FB}}$ aymmetry at $\mathrm{LHCb}$ as function of $m_{\mu \mu}$ at $7 \mathrm{TeV}$ (left) and $8 \mathrm{TeV}$ (right) [10].

\section{Measurement of $\sin ^{2} \theta_{\mathrm{eff}}^{\ell}$ at CMS}

CMS measured $\sin ^{2} \theta_{\text {eff }}^{\ell}$, using $\mathrm{Z}$ decays into electrons and muons collected at $8 \mathrm{TeV}$ during Run 1. The CMS measurement uses similar tools as the one at CDF and is based on a template fit of the $\cos \theta^{*}$ distributions. The asymmetry measurement is carried out in 6 bins for the dilepton rapidity $y_{\ell \ell}$ and in 12 bins for the dilepton invariant mass $m_{\ell \ell}$. The measured data and the results of the fit are shown in Fig. 5. Figure 6 shows instead the effect of PDF and $\sin ^{2} \theta_{\text {eff }}^{\ell}$ uncertainties on $A_{F B}$. The PDF uncertainties are estimated using NNNPDF 3.0 replicas [13]. The PDF uncertainties mainly affect $A_{F B}$ far from the $Z$ boson peak while $\sin ^{2} \theta_{\text {eff }}^{\ell}$ has the largest effect on the peak. By using weights proportional to $\exp \left(-\chi^{2} / 2\right)$, which is equivalent to profiling over the PDF replicas, the uncertainty due to PDF decreases from 0.00057 to 0.00030 .
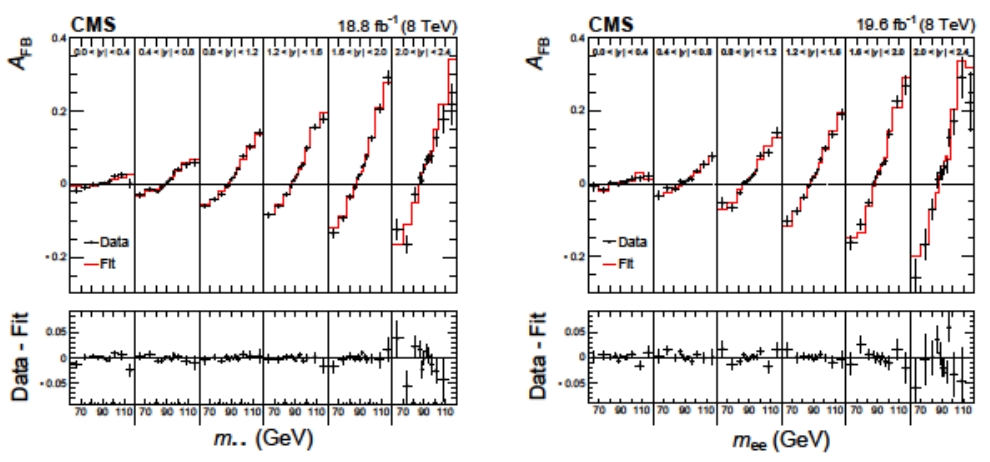

Figure 5: CMS measurement of $\mathrm{A}_{\mathrm{FB}}$. Left for $Z \rightarrow \mu \mu$. Righ for $Z \rightarrow e e$ [4].

The final CMS results [4] is:

$$
\begin{gathered}
\sin ^{2} \theta_{\text {eff }}^{\ell}=0.23101 \pm 0.00053= \\
=0.23101 \pm 0.00036 \text { (stat) } \pm 0.00018 \text { (syst) } \pm 0.00016 \text { (theory) } \pm 0.00031(\mathrm{PDF}) .
\end{gathered}
$$



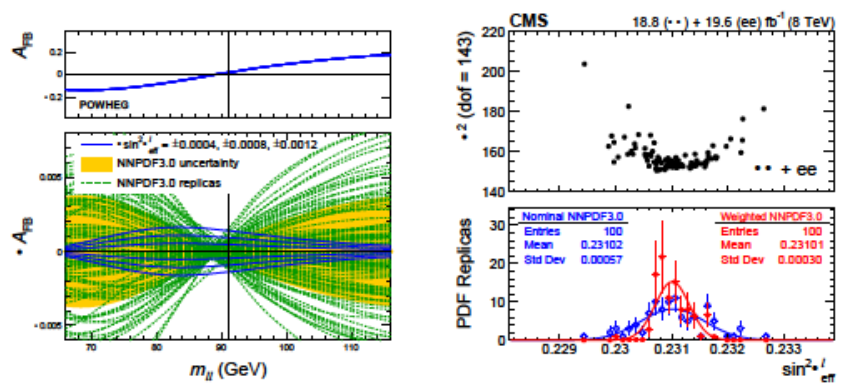

Figure 6: Effect of PDF and $\sin ^{2} \theta_{\mathrm{eff}}^{\ell}$ on $\mathrm{A}_{\mathrm{FB}}$ (left) and effect of the PDF weighting method (right) [4].

\section{Measurement of $\sin ^{2} \theta_{\mathrm{eff}}^{\ell}$ and triple differential distributions at ATLAS}

ATLAS previously carried out a measurement of $\sin ^{2} \theta_{\mathrm{eff}}^{\ell}$ using data collected at $7 \mathrm{TeV}$. The result is:

$$
\sin ^{2} \theta_{\mathrm{eff}}^{\ell}=0.23101 \pm 0.00053 \sin ^{2} \theta_{\mathrm{eff}}^{\ell}=0.2308 \pm 0.0005 \text { (stat) } \pm 0.0006 \text { (syst) } \pm 0.0009 \text { (PDF) }
$$

Their most recent analysis consists in a triple differential cross section measurement, differential in $m_{\ell \ell}, \cos \theta^{*}$ and $y_{\ell \ell}$ [2]. The analysis is carried our using $8 \mathrm{TeV}$ data in the $Z / \gamma \rightarrow \mu \mu$ and $Z / \gamma \rightarrow e e$ channels, where both leptons are central, and also in $Z / \gamma \rightarrow e e$ channel with one central and one forward electron. The analysis is performed in a total of 504+504+104 bins. Figure 7 shows the measurement as function of $y_{\ell \ell}$ in various $\cos \theta^{*}$ bins, positive and negative and for three different $m_{\ell \ell}$ ranges, below, on and above the $\mathrm{Z}$ peak. The data show good agreement with the predictions of POWHEG and these measurements can be used to extract the measurement of $\sin ^{2} \theta_{\mathrm{eff}}^{\ell}$.
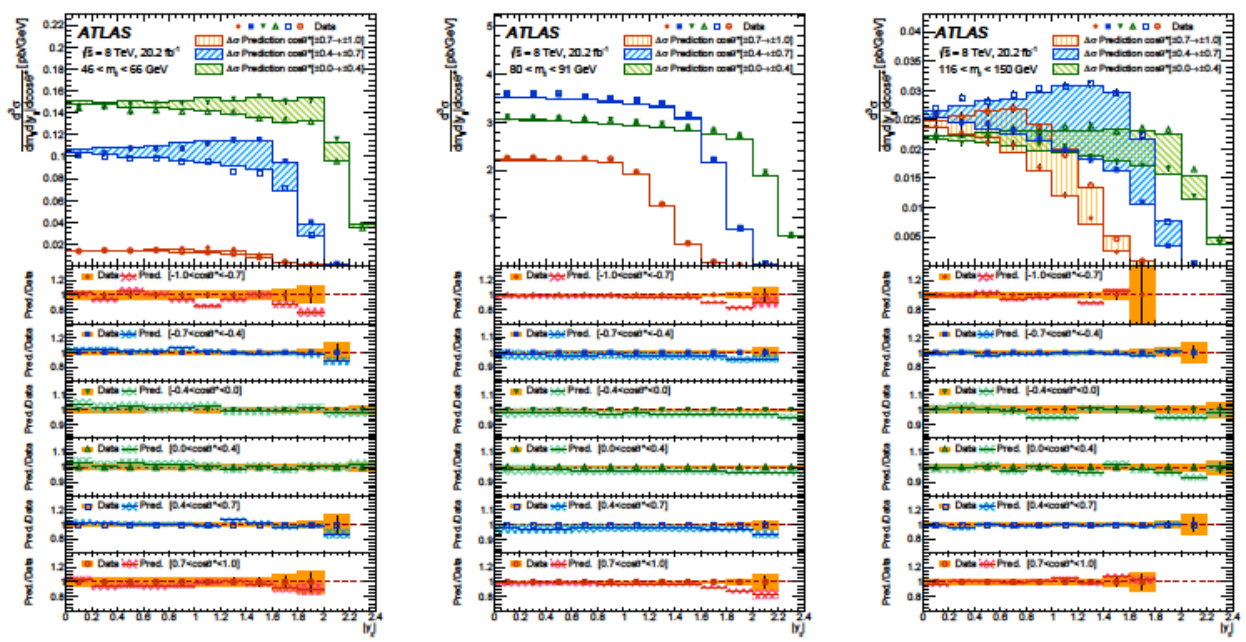

Figure 7: Measured differential cross sections at ATLAS as function of $y_{\ell \ell}$ in various $\cos \theta^{*}$ bins, positive and negative and for three different $m_{\ell \ell}$ ranges, below (left), on (central), and above the $\mathrm{Z}$ peak [2].

\section{Measurement of $Z$ boson angular coefficients at ATLAS and CMS}

Using Z decays also the Drell-Yan angular coeffcients have been measured at CDF[14] and 
LHC. The Drell-Yan cross section differential in the polar and azimuthal angles in the $Z$ boson rest frame is given by:

$$
\begin{gathered}
\frac{\mathrm{d}^{2} \sigma}{\mathrm{d} \cos \theta^{*} \mathrm{~d} \phi^{*}} \propto\left[\left(1+\cos ^{2} \theta^{*}\right)+A_{0} \frac{1}{2}\left(1-3 \cos ^{2} \theta^{*}\right)+A_{1} \sin \left(2 \theta^{*}\right) \cos \phi^{*}+A_{2} \frac{1}{2} \sin ^{2} \theta^{*} \cos \left(2 \phi^{*}\right)\right. \\
\left.+A_{3} \sin \theta^{*} \cos \phi^{*}+A_{4} \cos \theta^{*}+A_{5} \sin ^{2} \theta^{*} \sin \left(2 \phi^{*}\right)+A_{6} \sin \left(2 \theta^{*}\right) \sin \phi^{*}+A_{7} \sin \theta^{*} \sin \phi^{*}\right] .
\end{gathered}
$$

CMS measured the first four coefficients $A_{1}$ to $A_{4}$ [15] using dimuon events. CMS results are shown in Fig. 8. ATLAS measured all the angular coefficients $A_{1}$ to $A_{7}$ using decays into muons and electrons, including those with one forward electron. ATLAS measurements use regularization and shown in Fig. 9.
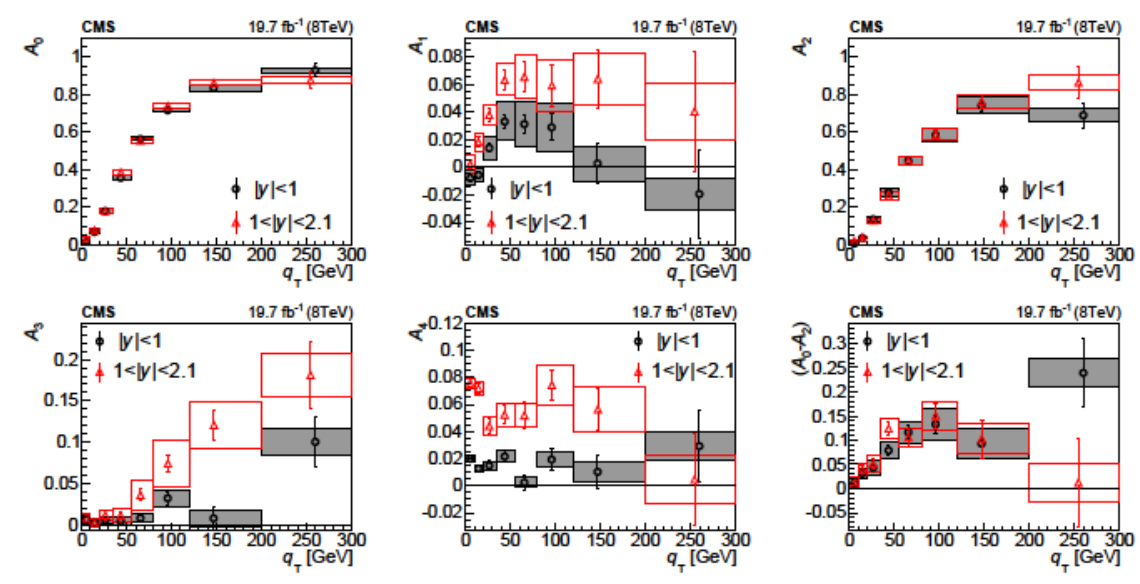

Figure 8: Angular coefficients measured at CMS [15].
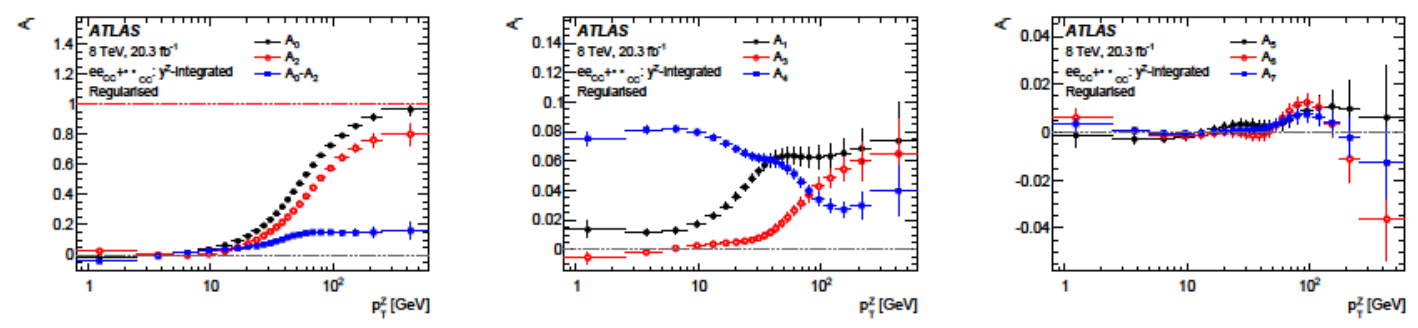

Figure 9: Angular coefficients measured by ATLAS as function of [16].

\section{Summary and Conclusions}

The summary of the reported $\sin ^{2} \theta_{\text {eff }}^{\ell}$ measurements is shown in table 1. After the LHCP conference, ATLAS presented a preliminary measurement of $\sin ^{2} \theta_{\text {eff }}^{\ell}=0.23140 \pm 0.00036$ [17], based on the measurement of the angular coefficients describing the $Z$ boson decay in $8 \mathrm{TeV}$ data [16] discussed in Section 7. CMS projections of sensitivity for the measurement of $\sin ^{2} \theta_{\mathrm{eff}}^{\ell}$ [18], shown in Fig. 10, indicate that at the end of LHC individual experiments should approach the precision of LEP and SLD and surpass it by the end of the high-luminosity LHC run. 


\begin{tabular}{|l|c|c|c|c|}
\hline Experiment & Int. lum. $\left(\mathrm{fb}^{-1}\right)$ & Central value & Stat. unc. & Syst. unc. \\
\hline ATLAS & 5 & 0.2308 & 0.0012 & 0.0005 \\
CMS & 21 & 0.23101 & 0.00052 & 0.00036 \\
Tevatron & 20 & 0.23148 & 0.00033 & 0.00027 \\
LHCb & 3 & 0.23142 & 0.0011 & 0.00073 \\
\hline
\end{tabular}

Table 1: Summary of the $\sin ^{2} \theta_{\text {eff }}^{\ell}$ measurements carried out at the hadron colliders at the time of the LHCP 2018 conference.

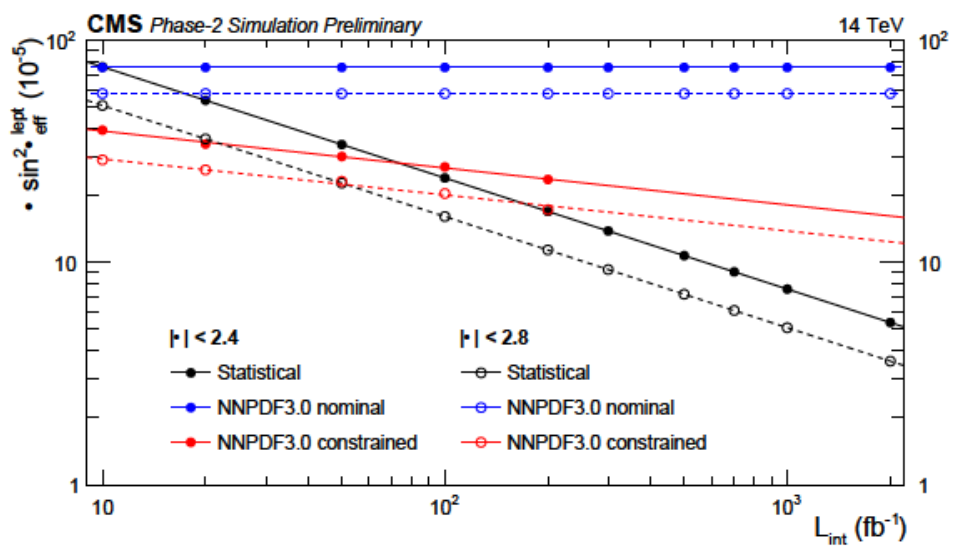

Figure 10: CMS Projected statistical, nominal PDF and constrained PDF uncertainties in $\sin ^{2} \theta_{\text {eff }}^{\ell}$ from the measurement of $A_{\mathrm{FB}}$ at $14 \mathrm{TeV} \mathrm{CM}$ energy as function of the integrated luminosities and for $|\eta|<2.4$ and $|\eta|<2.8$ acceptance selections for the muons. The nominal NNPDF3.0 uncertainty is calculated as a standard deviation of the extracted $\sin ^{2} \theta_{\text {eff }}^{\ell}$ over the 100 NNPDF3.0 replicas. The constrained NNPDF3.0 uncertainty is obtained using the weighting method discussed in the current CMS measurement [18].

\section{References}

[1] M. Baak et al. [Gfitter Group], Eur. Phys. J. C 74, 3046 (2014).

[2] M. Aaboud et al. [ATLAS Collaboration], JHEP 1712, 059 (2017).

[3] S. Schael et al. [ALEPH and DELPHI and L3 and OPAL and SLD Collaborations and LEP Electroweak Working Group and SLD Electroweak Group and SLD Heavy Flavour Group], Phys. Rept. 427, 257 (2006).

[4] A. M. Sirunyan et al. [CMS Collaboration], Eur. Phys. J. C 78, 701 (2018).

[5] D. Acosta et al. [CDF Collaboration], Phys. Rev. D 71, 052002 (2005).

[6] T. Aaltonen et al. [CDF Collaboration], Phys. Rev. D 88, 072002 (2013) Erratum: [Phys. Rev. D 88, 079905 (2013)].

[7] V. M. Abazov et al. [D0 Collaboration], Phys. Rev. Lett. 120, 241802 (2018).

[8] T. A. Aaltonen et al. [CDF and D0 Collaborations], Phys. Rev. D 97, 112007 (2018).

[9] A. Akhundov, A. Arbuzov, S. Riemann and T. Riemann, Phys. Part. Nucl. 45, 529 (2014).

[10] R. Aaij et al. [LHCb Collaboration], JHEP 1511, 190 (2015). 
[11] A. Bodek, Eur. Phys. J. C 67, 321 (2010).

[12] A. Bodek, J. Han, A. Khukhunaishvili and W. Sakumoto, Eur. Phys. J. C 76, 115 (2016).

[13] R. D. Ball et al. [NNPDF Collaboration], JHEP 1504, 040 (2015).

[14] T. Aaltonen et al. [CDF Collaboration], Phys. Rev. Lett. 106, 241801 (2011).

[15] V. Khachatryan et al. [CMS Collaboration], Phys. Lett. B 750, 154 (2015).

[16] G. Aad et al. [ATLAS Collaboration], JHEP 1608, 159 (2016).

[17] ATLAS collaboration, ATLAS-CONF-2018-037, http://cds.cern.ch/record/2630340.

[18] CMS Collaboration, CMS-PAS-FTR-17-001, http://cds.cern.ch/record/2294888. 